Z Epileptol 2018 - 31:282-295 https://doi.org/10.1007/s10309-018-0217-7 Online publiziert: 9 . November 2018 (c) Springer Medizin Verlag GmbH, ein Teil von Springer Nature 2018

CrossMark

Robert S. Fisher ${ }^{1,20} \cdot$ J. Helen Cross ${ }^{2} \cdot$ Carol D'Souza $^{3} \cdot$ Jacqueline A. French $^{4}$. Sheryl R. Haut ${ }^{5}$. Norimichi Higurashi ${ }^{6} \cdot$ Edouard Hirsch $^{7} \cdot$ Floor E. Jansen ${ }^{8}$. Lieven Lagae ${ }^{9}$. Solomon L. Moshé ${ }^{10}$. Jukka Peltola ${ }^{11}$ - Eliane Roulet Perez ${ }^{12}$. Ingrid E. Scheffer ${ }^{13}$. Andreas Schulze-Bonhage ${ }^{14}$. Ernest Somerville ${ }^{15}$. Michael Sperling ${ }^{16}$. Elza Márcia Yacubian ${ }^{17}$. Sameer M. Zuberi ${ }^{18,19}$ für die ILAE Commission for Classification and Terminology

'Stanford Department of Neurology \& Neurological Sciences, Stanford, USA; ${ }^{2}$ UCL-Institute of Child Health, Great Ormond Street Hospital for Children, London, Großbritannien; ${ }^{3}$ Bombay Epilepsy Society, Mumbai, Indien; ${ }^{4}$ Department of Neurology, NYU Langone School of Medicine, New York, USA; ${ }^{5}$ Montefiore Medical Center, Albert Einstein College of Medicine, New York, USA; ${ }^{6}$ Department of Pediatrics, Jikei University School of Medicine, Tokyo, Japan; ${ }^{7}$ Unite Francis Rohmer, Strasbourg, Frankreich; ${ }^{8}$ Department of Pediatric Neurology, Brain Center Rudolf Magnus, University Medical Center, Utrecht, Niederlande; ${ }^{9}$ Pediatric Neurology, University Hospitals KU Leuven, Leuven, Belgien; ${ }^{10}$ Saul R. Korey Department of Neurology, Department of Pediatrics, Dominick P. Purpura Department Neuroscience, Montefiore Medical Center, New York, USA; "Department of Neurology, Tampere University Hospital, Tampere, Finnland; ${ }^{12}$ Pediatric Neurorehabilitation Unit, CHUV, Lausanne, Schweiz; ${ }^{13}$ Austin Health and Royal Children's Hospital, Florey Institute and University of Melbourne, Melbourne, Australien; ${ }^{14}$ Epilepsy Center, University Medical Center Freiburg, Freiburg, Deutschland; ${ }^{15}$ Faculty of Medicine, Prince of Wales Clinical School, University of New South Wales, Sydney, Australien; ${ }^{16}$ Department of Neurology, Jefferson Comprehensive Epilepsy Center, Thomas Jefferson University, Philadelphia, USA; ${ }^{17}$ Department of Neurology and Neurosurgery, Epilepsy Research and Treatment Unit, São Paulo, Brasilien; ${ }^{18}$ The Paediatric Neurosciences Research Group, Royal Hospital for Children, Glasgow, Großbritannien; ${ }^{19}$ College of Medicine, Veterinary \& Life Sciences, University of Glasgow, Glasgow, Großbritannien; ${ }^{20}$ Neurology, SNHC, Palo Alto, USA

\title{
Anleitung (,instruction manual“) zur Anwendung der operationalen Klassifikation von Anfallsformen der ILAE 2017
}

ohne Expertenwissen nicht verstanden, einschließlich „dyskognitiv“, „psychisch“, „partiell“, „einfach-partiell“ und „komplex-partiell“. Für Nichtklinker kann es verwirrend sein, zu beurteilen, ob eine Person während eines Anfalls ein gestörtes Bewusstsein hat. Die neue Klassifikation behandelt diese relevanten Themen. Der folgende Text erläutert, wie die Klassifikation von Anfallsformen von 2017 anzuwenden ist.

\section{Methoden}

Die Klassifikation eines Anfalls beginnt mit der anamnestischen Erhebung oder Beobachtung bestimmter Symptome (manchmal als Anfallssemiologie bezeichnet), die bekannterweise bei üblichen Anfällen vorkommen. Die Haupt- symptome können nicht in einer Einszu-Eins-Beziehung Anfallsformen zugeordnet werden, da einige Symptome bei mehr als einer Anfallsform vorkommen. Ein Innehalten kann beispielsweise sowohl bei fokalen Anfällen mit gestörtem Bewusstsein (= nicht bewusst

Ad-hoc-Taskforce der DGfE, ÖGfE und der Schweizerischen Epilepsie-Liga (alphabetisch)

- Prof. Dr. Hajo Hamer, MHBA, Erlangen, Deutschland

- Dr. med. Günter Krämer, Zürich, Schweiz

- Prof. Dr. med. Stephan Rüegg, Basel, Schweiz

- Prof. Dr. med. Andreas Schulze-Bonhage Freiburg, Deutschland

- Univ. Prof. Dr. Mag. Eugen Trinka, FRCP, Salzburg, Österreich 


\section{Kernpunkte}

- Die ILAE stellt eine revidierte einfache und erweiterte Klassifikation von Anfallsformen bereit mit initialer Einteilung in einen fokalen, generalisierten oder unbekannten Anfallsbeginn.

- Fokale Anfälle werden optional unterteilt in solche mit erhaltenem oder gestörtem Bewusstsein (bewusst erlebt oder nicht bewusst erlebt). Spezifische motorische oder nichtmotorische Klassifikatoren können hinzugefügt werden.

- Anfälle mit generalisiertem Anfallsbeginn können motorisch, tonisch-klonisch, klonisch, tonisch, myoklonisch, myoklonisch-tonisch-klonisch, myoklonisch-atonisch, atonisch und epileptische Spasmen sein.

- Anfälle mit generalisiertem Anfallsbeginn können auch nichtmotorisch (Absencen) sein: typische Absence, atypische Absence, myoklonische Absence oder Absence mit Lidmyoklonien.

- Zusätzliche Deskriptoren und Freitext zur Anfallscharakterisierung werden befürwortet. Eine Gegenüberstellung alter und neuer Termini soll die Akzeptanz der neuen Klassifikation erleichtern.

erlebten fokalen Anfällen) als auch bei Absencen auftreten. Tonisch-klonische Aktivität kann bei einem generalisierten Anfall von Beginn an oder im Verlauf der Entwicklung eines Anfalls mit fokalem Anfallsursprung auftreten. Umgekehrt kann eine Anfallsform oft mit mehreren Symptomen einhergehen. Die Bezeichnung einer Anfallsform als „Anfall mit Automatismen" gestattet keine Unterscheidung zwischen einem fokalen Anfall mit gestörtem Bewusstsein und einer Absence. Da diese beiden Anfallsformen unterschiedlich behandelt werden und unterschiedliche Prognosen haben, ist die Beibehaltung unterschiedlicher Anfallsformen nützlich, selbst wenn zur Klassifikation ein gewisser Grad an Interpretation jenseits der direkten Beobachtung erforderlich sein kann. Die Unterscheidung von Anfallsformen kann üblicherweise anhand der Erkennung einer charakteristischen Abfolge von Symptomen und anderen klinischen Beobachtungen vorgenommen werden. Typische Absencen zeigen beispielsweise eine schnellere funktionelle Erholung als fokale Anfälle mit Bewusstseinsstörung. Manchmal sind zusätzliche Informationen durch Elek-

\section{Basisversion der 2017er-ILEA-Anfallsklassifikation ${ }^{\mathrm{a}}$}

fokaler Beginn

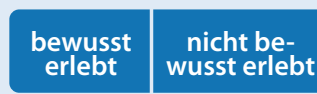

motorischer Beginn

nichtmotorischer Beginn

fokal zu bilateral

tonisch-klonisch generalisierter Beginn

motorisch

- tonisch-klonisch

- anders motorisch

nichtmotorisch

(Absence) unbekannter Beginn

motorisch

- tonisch-klonisch

- anders motorisch

nichtmotorisch

(Absence)

nicht klassifiziert

Abb. 1 A Die Basisversion der 2017er-ILAE-Klassifikation der Anfallsformen. ${ }^{2}$ Definitionen, andere Anfallsformen und Deskriptoren sind in einer Begleitpublikation und im Glossar der Fachbegriffe aufgeführt. ${ }^{\mathrm{b}}$ Aufgrund unzureichender Information oder fehlender Möglichkeit, den Anfall anderen Kategorien zuzuordnen

troenzephalographie (EEG), Bildgebung oder Laboruntersuchungen erforderlich, um einen Anfall korrekt zu klassifizieren. In diesen Fällen beginnt sich die Anfallsklassifikation unmerklich mit der Diagnose des Epilepsiesyndroms zu vermischen $[1,2]$. In Anbetracht eines Mangels an einem fundamentalen pathophysiologischen Verständnis der unterschiedlichen Anfallsmanifestationen spiegelt die Symptomgruppierung in Anfallsformen eine operationelle Meinung darüber wider, welche Gruppierungen hinreichend unterschiedlich und verbreitet sind, um einen spezifischen Namen zu rechtfertigen [3]. Diese Klassifikation wurde für die klinische Praxis entwickelt, sie kann jedoch auch von Forschern und anderen Gruppen mit spezifischen Zielen verwendet werden.

\section{Ergebnisse}

Die Anfallsklassifikation der ILAE von 2017 bietet in Abhängigkeit von dem gewünschten Grad an Detailliertheit eine einfache und eine erweiterte Versionen an. Die einfache Version stimmt prinzipiell mit der erweiterten überein, jedoch mit vereinfachten Subkategorien.

\section{Einfache Klassifikation}

- Abb. 1 zeigt die einfache Klassifikation. Anfälle werden zunächst anhand ihres Ursprunges eingeteilt. Anfälle mit fokalem Beginn sind definiert als „mit Ursprung in Netzwerken, die auf eine
Hemisphäre begrenzt sind. Er kann umschrieben lokalisiert oder weiter ausgedehnt sein. Fokale Anfälle können in subkortikalen Strukturen beginnen."Anfälle mit generalisiertem Anfallsbeginn ,entstehen an irgendeinem Punkt eines bilateralen Netzwerkes und breiten sich hierin rasch aus" [4]. Anfälle unbekannten Ursprungs können ebenfalls definierende motorische (z.B. tonisch-klonische) oder nichtmotorische (z.B. Innehalten) Charakteristika haben. Mit weiteren Anfallsbeobachtungen kann im Verlauf eine Reklassifizierung von Anfällen mit unbekanntem Ursprung zu solchen mit fokalem oder generalisiertem Beginn möglich werden. Entsprechend ist „Anfall mit unbekanntem Anfallsursprung "kein Anfallscharakteristikum, sondern Ausdruck eines unzureichenden Kenntnisstandes. Wenn eine Anfallsform als "fokal", „generalisiert" oder ,unbekannt" bezeichnet wird, ist der Ursprung gemeint.

Optional kann eine detailliertere Klassifikation erfolgen. Die nächste Stufe der Klassifikation fokaler Anfälle erfolgt anhand der Bewusstheit. Bewusstes Erleben ist operational definiert als Wissen um das Selbst und die Umgebung. Die Erfassung des bewussten Erlebens ist ein pragmatischer Surrogatmarker für die Bestimmung, ob das Bewusstsein gestört ist. Während eines bewusst erlebten fokalen Anfalls ist das Bewusstsein erhalten. Diese Bewusstheit bezieht sich auf die Bewusstseinslage während des Anfalls, nicht auf das Wissen, ob sich ein Anfall ereignet hat. Wenn das Bewusstsein 
Z Epileptol 2018·31:282-295 https://doi.org/10.1007/s10309-018-0217-7

(c) Springer Medizin Verlag GmbH, ein Teil von Springer Nature 2018

R. S. Fisher · J. H. Cross · C. D'Souza · J. A. French · S. R. Haut · N. Higurashi · E. Hirsch · F. E. Jansen - L. Lagae · S. L. Moshé · J. Peltola · E. Roulet Perez - I. E. Scheffer · A. Schulze-Bonhage · E. Somerville · M. Sperling · E. M. Yacubian · S. M. Zuberi für die ILAE Commission for Classification and Terminology

\section{Anleitung (,instruction manual“') zur Anwendung der operationalen Klassifikation von Anfallsformen der ILAE 2017}

\section{Zusammenfassung}

Dieser Begleittext zur Einführung der Klassifikation von Anfallsformen durch die Internationale Liga gegen Epilepsie (ILAE) bietet eine Anleitung, wie diese Klassifikation anzuwenden ist. Die Klassifikation wird anhand von Tabellen, einem Glossar wichtiger Fachbegriffe, einer tabellarischen Zuordnung alter und neuer Fachbegriffe, empfohlenen Abkürzungen und anhand von Beispielen verdeutlicht. Abhängig von der gewünschten Detailgenauigkeit kann eine einfache und eine ausführliche Version der Klassifikation benutzt werden. Die semiologischen Hauptsymptome werden als Grundlage zur Kategorisierung von Anfällen verwendet, die einen fokalen, generalisierten oder unbekannten Ursprung haben können. Alle fokalen Anfälle können zusätzlich hinsichtlich der erhaltenen oder gestörten Bewusstheit als bewusst oder nicht bewusst erlebt charakterisiert werden. Hierbei bedingt eine Beeinträchtigung in irgendeinem Abschnitt des Anfalls eine Klassifikation als Anfall mit Bewusstseinsstörung. Fokale Anfälle können ferner optional durch die ersten motorischen Zeichen als atonisch, mit Automatismen, klonisch, als epileptische Spasmen, hyperkinetisch, myoklonisch oder tonisch charakterisiert werden. Anfälle mit nichtmotorischem Beginn können sich als autonom, mit Innehalten (Verhaltensarrest), kognitiv, emotional oder sensorisch manifestieren. Die erste prominente Manifestation definiert die Anfallsform, die sich dann zu anderen Symptomen weiterentwickeln kann. Fokale Anfälle können sich zu bilateral tonisch-klonischen Anfällen entwickeln. Generalisierte Anfälle beinhalten eine frühe Involvierung bilateraler Netzwerke von Anfang an. Generalisierte motorische Anfallscharakteristika umfassen atonische, klonische, myoklonische, myoklonischatonische, myoklonisch-tonisch-klonische, tonische, tonisch-klonische Manifestationen oder epileptische Spasmen. Nichtmotorische Anfälle (Absencen) sind typisch oder atypisch, oder Anfälle mit prominenter myoklonischer Aktivität oder Lidmyoklonien. Anfälle unbekannten Ursprungs können Eigenschaften haben, die immerhin als motorisch, nichtmotorisch, tonisch-klonisch, epileptische Spasmen oder als Innehalten klassifiziert werden können. Diese Anleitung zur Anfallsklassifikation der ILAE 2017 kann die Akzeptanz der neuen Systematik unterstützen.

Schlüsselwörter Klassifikation · Anfälle · Fokal · Generalisiert . Epilepsie (Taxonomie)

\section{Instruction manual for the ILAE 2017 operational classification of seizure types}

\section{Abstract}

This companion paper to the introduction of the International League Against Epilepsy (ILAE) 2017 classification of seizure types provides guidance on how to employ the classification. The classification is illustrated by tables, a glossary of relevant terms, a table mapping old to new terms, recommended abbreviations and examples. Basic and extended versions of the classification are available depending on the desired degree of detail. Key signs and symptoms of seizures (semiology) are used as a basis for categorization of seizures that are focal or generalized from onset or with unknown onset. Any focal seizure can be further optionally characterized with respect to whether awareness is retained or impaired. Impaired awareness during any segment of the seizure renders it classified as a focal impaired awareness seizure. Focal seizures can be further optionally characterized by the first motor signs and symptoms as atonic, automatisms, clonic, epileptic spasms, hyperkinetic, myoclonic or tonic activity. Nonmotor onset seizures can be manifested as autonomic, behavior arrest, cognitive, emotional, or sensory dysfunction. The earliest prominent manifestation defines the seizure type, which can then further progress to other signs and symptoms. Focal seizures can become bilateral tonic-clonic seizures. Generalized seizures include an early involvement of bilateral networks from the onset. Generalized motor seizure characteristics comprise atonic, clonic, epi- leptic spasms, myoclonic, myoclonic-atonic, myoclonic-tonic-clonic, tonic or tonic-clonic manifestatíons. Nonmotor (absence) seizures are typical or atypical or seizures that present prominent myoclonic activity or eyelid myoclonia. Seizures of unknown origin may have features that can still be classified as motor, nonmotor, tonic-clonic, epileptic spasms or behavior arrest. This users' manual for the ILAE 2017 seizure classification can assist the acceptance of the new system.

Keywords Classification · Seizures · Focal · Generalized · Epilepsy (taxonomy) während irgendeines Anfallsabschnittes beeinträchtigt ist, wird der Anfall als nicht bewusst erlebter fokaler Anfall klassifiziert. Praktisch bedeutet dies, dass ein Patient das bewusste Erleben im Anfall (durch Schilderung der Umstände des Anfalls und seines Ablaufes) belegen kann. Manche Anfälle können eine transiente epileptische Amnesie [5] mit erhaltenem Bewusstsein erzeugen, aber die Klassifikation solcher Anfälle würde eine außergewöhnlich exakte Dokumentation durch Beobachter erfordern. Man kann hierfür die Formulierung „nicht bewusst erlebter fokaler Anfall“ benutzen. Hierbei ist wichtig, dass auch eine unvoll- ständige Beeinträchtigung des Bewusstseins vorliegen kann.

Reagibilität ist ein hiervon zu unterscheidendes klinisches Merkmal, das erhalten oder gestört sein kann bei Anfällen mit oder ohne erhaltenes Bewusstsein. Obwohl Reagibilität ein wichtiger beschreibender Aspekt von Anfällen ist, 
Erweiterte Version der 2017er-ILEA-Anfallsklassifikation ${ }^{a}$

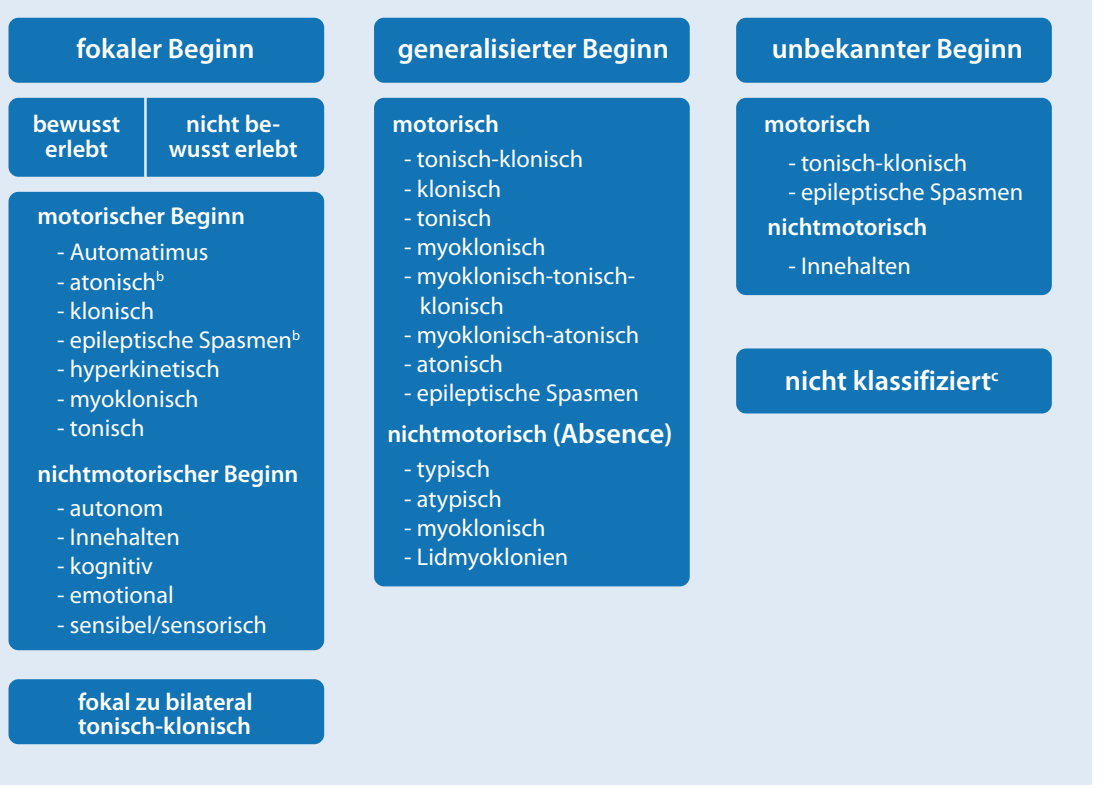

Abb. 2 A Die erweiterte operationale 2017er-ILAE-Klassifikation der Anfallsformen. Die folgenden Erklärungen sollen eine Anleitung bei der Wahl der Anfallsform sein. Bei fokalen Anfällen ist die Angabe der Bewusstseinslage bzw. des bewussten oder nicht bewussten Erlebens optional. Bewusstes Erleben bedeutet, dass der Patient sich seiner selbst und der Umgebung gewahr ist, auch wenn er sich nicht bewegen kann. Ein bewusst erlebter fokaler Anfall entspricht dem früheren Begriff „einfach-partieller Anfall“. Ein nicht bewusst erlebter Anfall entspricht dem früheren Begriff "komplex-partieller Anfall“ und ein beeinträchtigtes Bewusstsein während irgendeines Abschnittes des Anfalls bedingt die Einstufung als nicht bewusst erlebter Anfall. Bewusst erlebte oder nicht bewusst erlebte fokale Anfälle können darüber hinaus durch eines der unten angeführten initialen motorischen oder nicht-motorischen Symptome charakterisiert werden, die das erste prominente Anfallssymptom widerspiegeln. Anfälle sollten anhand des frühesten prominenten Zeichens klassifiziert werden, mit Ausnahme eines Innehaltens, das nur dann zur Klassifikation genutzt werden soll, wenn es während des gesamten Anfalls das prominente Symptom ist. Daneben kann bei der Bezeichnung eines fokalen Anfalls auf die Erwähnung des Bewusstseinszustandes verzichtet werden, wenn dies nicht anwendbar oder der Zustand nicht bekannt ist, und der Anfall unmittelbar durch seine initialen motorischen oder nicht-motorischen Charakteristika klassifiziert werden. So wird das Bewusstsein bei atonischen Anfällen und epileptischen Spasmen üblicherweise nicht spezifiziert. Kognitive Anfälle implizieren eine Beeinträchtigung der Sprache oder anderer kognitiver Bereiche oder positive Symptome wie Déjà vu, Halluzinationen, Illusionen oder Wahrnehmungsstörungen. Emotionale Anfälle umfassen Angst, Furcht, Freude, andere Emotionen oder das Auftreten von scheinbar affektivem Verhalten ohne subjektiv erlebte Emotionen. Eine Absence ist atypisch bei langsamem Beginn oder Ende oder bei signifikanten Veränderungen des Muskeltonus, begleitet von atypischer, langsamer, generalisierter Spike-Wave-Aktivität im EEG. Ein Anfall kann aufgrund mangelhafter Informationen unklassifiziert sein oder weil man die Form nicht in die anderen Kategorien einordnen kann. 'Definitionen, andere Anfallsformen und Deskriptoren sind in einer Begleitpublikation und im Glossar der Fachbegriffe aufgeführt. ber Bewusstseinsgrad wird üblicherweise nicht spezifiziert. 'Aufgrund unzureichender Information oder fehlender Möglichkeit, den Anfall anderen Kategorien zuzuordnen

wird sie in der Klassifikation der ILAE von 2017 nicht zur Beschreibung spezifischer Anfallsformen verwendet. Die basale Klassifikation erlaubt ferner, Anfälle in solche mit motorischem oder nichtmotorischem Beginn (z. B. sensorischen Symptomen) zu klassifizieren. Die weitere Spezifizierung nutzt die im Folgenden diskutierte erweiterte Klassifikation. kation wird „bilateral“ für die Ausbreitung von fokalen Anfällen und „generalisiert" für Anfälle mit generalisiertem Beginn verwendet.

Anfälle mit generalisiertem Beginn werden in motorische und nichtmotorische (Absence-)Anfälle unterteilt. Die Bewusstseinslage wird nicht als Klassifikator generalisierter Anfälle benutzt, da die überwiegende Mehrzahl generalisierter Anfälle (wenn auch nicht alle) mit einer Bewusstseinsstörung einhergehen. Definitionsgemäß sollte motorische Aktivität von Beginn an bilateral sein, aber in der basalen Klassifikation muss die motorische Aktivität nicht spezifiziert werden. Bei asymmetrischem Beginn der motorischen Aktivität kann es in der Praxis schwierig sein festzulegen, ob ein Anfall einen fokalen oder generalisierten Beginn hat.

Absencen (das Präfix „mit generalisiertem Beginn“ ist implizit) präsentieren sich durch ein plötzliches Sistieren von Aktivität und Bewusstsein. Absencen kommen v. a. in jüngeren Altersgruppen vor und zeigen meist weniger komplexe Automatismen als fokale Anfälle mit gestörtem Bewusstsein, aber die Abgrenzung ist unscharf. EEG-Informationen können zur korrekten Klassifikation erforderlich sein. Fokale epileptische Aktivität kann bei fokalen Anfällen gesehen werden und bilateral synchrone Spikewave-Entladungen bei Absencen.

\section{Erweiterte Klassifikation}

Die erweiterte Klassifikation (• Abb. 2) bietet eine zusätzliche Ebene von Anfallsbezeichnungen, die auf der einfachen Klassifikation aufbaut. Die vertikale Organisation der Kategorie von Anfällen fokalen Ursprungs ist nicht hierarchisch, da die Angabe des Bewusstseinszustandes optional ist. Ein fokaler Anfall kann als „bewusst erlebt fokal" (korrespondierend zur Bezeichnung ,einfach-partieller Anfall“ von 1981) oder als "nicht bewusst erlebt fokal" (korrespondierend zur Bezeichnung „komplex-partieller Anfall" von 1981) klassifiziert werden. Fokale Anfälle mit erhaltenem oder gestörtem Bewusstsein können optional durch zusätzliche Angabe eines initialen motorischen oder nichtmotorischen 
Zeichens klassifiziert werden, die das früheste prominente Symptom außer der Bewusstseinslage widerspiegeln. Alternativ kann bei einem fokalen Anfall die Angabe zur Bewusstseinslage entfallen, wenn diese nicht anwendbar oder unbekannt ist und der fokale Anfall direkt durch die ersten motorischen oder nichtmotorischen Zeichen charakterisiert wird.

Bei fokal beginnenden Anfällen sollte der Arzt den Grad an Bewusstheit, wie in der einfachen Klassifikation beschrieben, beurteilen. Man sollte die Patienten fragen, ob sie Vorkommnisse während der Anfälle bewusst erlebt haben, selbst wenn sie nicht reagiert haben oder nicht in der Lage waren, Sprache zu verstehen, oder ob dies nicht der Fall war. Wenn jemand den Raum während des Anfalls betrat, erinnert er sich danach daran? Eine Fremdanamnese kann bei der Klärung des Verhaltens im Anfall helfen. Der Versuch, zwischen iktualem und postiktualem Zustand zu unterscheiden, ist sehr wichtig, da das Bewusstsein während Letzterem zurückkehrt. Wenn der Bewusstseinszustand sicher ist, wie beispielsweise häufig bei atonischen Anfällen und epileptischen Spasmen, können Anfälle als fokal klassifiziert werden, ohne den Bewusstseinszustand zu spezifizieren. Beschreibungen des Bewusstseinsgrades sind optional und werden lediglich durchgeführt, insofern er bekannt ist. Ein „bewusst erlebter fokaler Anfall“, ob mit oder ohne weitergehende Charakterisierung, entspricht dem alten Begriff „einfach partieller Anfall“, und „nicht bewusst erlebter fokaler Anfall“ entspricht dem alten Begriff „komplex-partieller Anfall“. Folgende Begriffe in der „fokalen“ Gruppe der erweiterten Klassifizierung können weitergehend den Typ von fokalen Anfällen ohne oder mit Bewusstseinsbeeinträchtigung spezifizieren. Alternativ kann der Grad an Bewusstheit unspezifiziert bleiben und ein Anfall als fokaler Anfall mit einem der motorischen oder nichtmotorischen Anfangscharakteristika, wie in - Tab. 2 angeführt, klassifiziert werden.

Fokal beginnende Verhaltensweisen umfassen folgende Symptome: atonisch (fokaler Tonusverlust), tonisch (anhaltende fokale Versteifung), klonisch (foka- les rhythmisches Zucken), myoklonisch (unrhythmisches, kurzes fokales Zucken) oder epileptische Spasmen (fokale Flexion oder Extension der Arme und Flexion des Rumpfes). Die Unterscheidung zwischen klonisch und myoklonisch ist etwas arbiträr, aber klonisch impliziert durchgehende, stereotype Zuckungen in regelmäßigen Abständen, während Myoklonien weniger regelmäßig und in kürzeren Gruppen auftreten. Andere, weniger offensichtlich fokale motorische Verhaltensweisen beinhalten hyperkinetische (tretende, schlagende) Aktivitäten und Automatismen.

Ein Automatismus ist eine mehr oder weniger koordinierte, ziellose, repetitive motorische Aktivität. Beobachter sollten gefragt werden, ob der Patient repetitive, ziellose Verhaltensfragmente aufzeigte, die unter anderen Umständen als normal erscheinen könnten. Manche Automatismen überschneiden sich mit anderem motorischem Verhalten, beispielsweise „radfahrenden“ Bewegungsschablonen oder hyperkinetischer Aktivität, weswegen die Klassifizierung mehrdeutig wird. Die 2017er-ILAE-Klassifizierung ordnet arbiträr radfahrende Bewegungen den hyperkinetischen Anfällen statt Anfällen mit Automatismen zu. Automatismen können bei fokalen Anfällen und bei Absencen auftreten.

Bei einem fokal motorischen Anfall mit Innehalten sistiert die Bewegung und fehlt eine Reaktivität. Da ein kurzes Innehalten zu Anfallsbeginn häufig und schwer feststellbar ist, sollte ein „fokaler Anfall mit Innehalten“ diesen Arrest als prädominanten Aspekt des gesamten Anfalls beinhalten.

Fokale autonome Anfälle zeigen sich in Form gastrointestinaler Empfindungen, einem Gefühl von Hitze oder Kälte, Hitzewallungen, Piloarrektionen (Gänsehaut), Palpitationen, sexueller Erregung, respiratorischen Veränderungen oder anderen autonomen Effekten. Fokale kognitive Anfälle können festgestellt werden, wenn der Patient während Anfällen von sprachlichen Defiziten, Beeinträchtigungen des Denkens oder assoziierter höherer kortikaler Funktionen berichtet oder diese zeigt und wenn diese Symptome gegenüber anderen Anfallsmanifestationen im Vordergrund stehen.
Déjà vu, Jamais vu, Halluzinationen, Illusionen und zwanghaftes Denken sind Beispiele induzierter, abnormaler kognitiver Phänomene. Ein korrekterer, wenn auch weniger wohlklingender Begriff wäre „fokaler Anfall mit beeinträchtigter Kognition“, aber die Beeinträchtigung der Kognition kann unterstellt werden, da Anfälle niemals kognitive Funktionen verbessern. Fokale emotionale Anfälle können mit Stimmungsschwankungen, einschließlich Angst, Agitation, Wut, Wahn, Glücksempfindung, Freude, Ekstase, Lachen oder Weinen einhergehen. Manche dieser Phänomene sind subjektiv und müssen vom Patient oder Betreuer erinnert und berichtet werden. Emotionale Symptome umfassen eine subjektive Komponente, wohingegen anscheinend affektive Zeichen von subjektiver Emotionalität begleitet sein können oder auch nicht. Eine Beeinträchtigung des bewussten Erlebens von Ereignissen im Anfall klassifiziert den Anfall nicht als einen fokalen kognitiven Anfall, da Bewusstseinsstörungen bei jedem fokalen Anfall eintreten können. Ein fokaler sensorischer Anfall kann somatosensible, olfaktorische, visuelle, auditive, gustatorische, Temperaturempfindungen oder vestibuläre Empfindungen hervorrufen.

Der Arzt muss entscheiden, ob ein Ereignis ein einzelner Anfall mit sich entwickelnden Manifestationen im Verlauf ist oder alternativ 2 separaten Anfällen entspricht. Diese Unterscheidung kann manchmal schwierig sein. Eine reibungslose, kontinuierliche Entwicklung von Symptomen und EEG-Mustern (wo verfügbar) legt nahe, dass das Vorkommnis ein einziger Anfall ist. Die Wiederholung einer stereotypen Sequenz an Zeichen, Symptomen und EEG-Veränderungen $\mathrm{zu}$ unterschiedlichen Zeiten deutet auf eine einheitliche Anfallsform hin. Einheitliche fokale Anfälle werden nach der initialen Manifestation und der Integrität oder der Beeinträchtigung des Bewusstseins zu irgendeinem Zeitpunkt des Anfalls klassifiziert. Im Gegensatz hierzu weisen diskontinuierliche, unterbrochene oder nichtstereotype Ereignisse auf eine Klassifizierung als mehr als eine Anfallsform hin. Man betrachte ein Ereignis mit Déjà vu, repetitivem, grundlosem Schmatzen, Bewusstseins- 
verlust, forcierter Version nach rechts und Tonisierung des rechten Armes. Die gleichmäßige Entwicklung legt eine einheitliche Anfallsform nahe, die als nicht bewusst erlebter fokaler kognitiver Anfall klassifiziert werden würde. Es wäre nützlich, Informationen über die Entwicklung zu Automatismen und zur tonischen Version als optionale Beschreibung, nicht jedoch als Anfallsform, anzufügen. In einem anderen Szenario kann ein Arzt einen Anfall mit Angst und Bewusstseinsverlust antreffen. Der Patient erholt sich und hat 30 min später ein Ereignis mit Kribbeln im rechten Arm bei klarem Bewusstsein. Solch eine Sequenz zeigt 2 separate Anfälle, der erste ist ein nicht bewusst erlebter fokaler emotionaler Anfall und der zweite ein bewusst erlebter fokaler sensorischer Anfall.

Manchmal kommen andere fokale Anfallsformen vor, z. B. fokale tonischklonische Anfälle, jedoch nicht häufig genug, um diese als eigenen Anfallstyp zu benennen. Anstatt die Bezeichnung "sonstige“ in jede Kategorie zu integrieren, wurde entschieden, zur unspezifischen Anwendung der höheren Kategorie zurückzugehen, so wie motorischer Beginn oder nichtmotorischer Beginn, falls die nächste Detailstufe unklar ist oder der Anfall nicht als eigene Anfallsform gelistet ist.

Die Klassifikation von Anfällen mit generalisiertem Beginn ähnelt der 1981er-Klassifikation, sie wurde nur um wenige neue Formen erweitert. Das Bewusstsein ist bei Anfällen mit generalisiertem Beginn meist beeinträchtigt, sodass der Grad an Bewusstheit nicht als Klassifikator für diese Anfälle genutzt wird. Die Haupteinteilung ist in motorische und nichtmotorische (Absence-)Anfallsformen. Die Termini „motorisch“ und „nicht-motorisch“ (Absence) werden benutzt, um die Charakterisierung von generalisiert beginnenden motorischen und nichtmotorischen Anfällen, über die sonst nichts gesagt werden kann, zu ermöglichen, wobei „motorisch“ und „nichtmotorisch“ (Absence) wegelassen werden kann, falls der Anfallsname unmissverständlich ist, wie z. B. bei "generalisierter tonischer Anfall“. Das Wort "generalisiert" kann für
Anfälle wie Absencen, die ausschließlich einen generalisierten Beginn haben, entfallen.

„Tonisch-klonisch“ bleibt die Bezeichnung, die „Grand mal“ ersetzt, obwohl die verbreitete Verwendung des alten französischen Begriffes zweifellos fortbestehen wird. Da es eine neue Anfallsform gibt, die durch Myoklonien vor einer Tonisierung (Versteifung) und Kloni (anhaltende rhythmische Zuckungen) charakterisiert ist, ist es wichtig, die frühen Bewegungen eines tonisch-klonischen Anfalls als tonisch zu dokumentieren. Die klonische Phase eines tonisch-klonischen Anfalls zeigt typischerweise eine regelmäßig abnehmende Frequenz der Kloni im Anfallsverlauf. Während eines tonisch-klonischen Anfalls kommt es vor oder gleichzeitig mit tonischen und klonischen Bewegungen zum Bewusstseinsverlust. Manche tonisch-klonischen Anfälle führen zu einem unspezifischen Gefühl eines nahenden Anfalls oder einer kurzen Periode einer Kopf- oder Rumpfversion, dies stellt jedoch einen generalisierten Beginn nicht infrage, da biologische Prozesse nie perfekte Synchronizität zeigen. Der Arzt muss beurteilen, ob tatsächlich ein fokaler Beginn vorliegt.

Generalisierte klonische Anfälle beginnen, entwickeln sich zu und enden mit rhythmischen beidseitiger Zuckungen der Extremitäten sowie häufig von Kopf, Nacken, Gesicht und Rumpf. Generalisierte klonische Anfälle kommen viel seltener vor als tonisch-klonische Anfälle, üblicherweise bei Kindern, und sollten von Zittrigkeit und Schauderattacken unterschieden werden [6].

Generalisierte tonische Anfälle manifestieren sich als bilaterale Versteifung oder Elevation der Gliedmaßen, häufig mit Versteifung des Nackens. Die Klassifizierung geht davon aus, dass auf die tonische Aktivität keine klonischen Bewegungen folgen. Die tonische Aktivität kann eine anhaltende abnormale Körperhaltung sein, entweder in Streckung oder Beugung, manchmal begleitet von Zittern der Extremitäten. Die Tonisierung kann schwer von einer Dystonie zu unterscheiden sein, definiert als anhaltende Kontraktion von agonistischen und antagonistischen Muskeln, die athetoide oder drehende Bewegungen bewirkt, die bei längerer Dauer zu abnormalen Körperhaltungen führen.

Generalisierte myoklonische Anfälle können isoliert oder in Verbindung mit tonischer oder atonischer Aktivität auftreten. Myoklonien unterscheiden sich von Kloni durch eine kürzere Dauer und fehlende Rhythmizität. Das Symptom einer Myoklonie kann epileptische und nichtepileptische Ursachen haben.

Generalisierte myoklonisch-tonischklonische Anfälle beginnen mit wenigen myoklonischen Zuckungen, gefolgt von tonisch-klonischen Phänomenen. Diese Anfälle sind häufig bei Patienten mit juveniler myoklonischer Epilepsie [7], gelegentlich auch bei anderen generalisierten Epilepsien. Es ist strittig, ob die ersten Zuckungen myoklonisch oder klonisch sind, aber selten sind sie hinreichend anhaltend, um sie klonisch zu nennen.

Ein myoklonisch-atonischer Anfall geht mit kurzen Zuckungen der Gliedmaßen oder des Rumpfes einher, gefolgt von einem schlaffen Sturz. Diese Anfälle, früher myoklonisch-astatisch genannt, werden am häufigsten beim DooseSyndrom beobachtet [8], können aber auch beim Lennox-Gastaut-Syndrom und anderen Syndromen vorkommen.

„Atonisch“ bedeutet ohne Muskeltonus. Bei einem Tonusverlust der Beine während eines generalisierten atonischen Anfalls stürzt der Patient auf das Gesäß oder manchmal vorwärts auf Knie und Gesicht. Die Erholung erfolgt meistens innerhalb von Sekunden. Im Gegensatz hierzu führen tonische oder tonisch-klonische Anfälle typischerweise zu einem Sturz nach hinten.

Epileptische Spasmen wurden früher „infantile Spasmen“ genannt; diese Bezeichnung bleibt angemessen für epileptische Spasmen, die während des Kindesalters auftreten. Ein epileptischer Spasmus zeigt sich in Form einer plötzlichen Flexion oder Extension oder einer Mischung von Extension und Flexion vorrangig der proximalen Extremitäten und Rumpfmuskulatur. Spasmen treten häufig in Clustern und bevorzugt in der Kindheit auf.

Generalisierte nichtmotorische Anfallsformen umfassen unterschiedliche Typen von Absencen. Die Taskforce behielt die Unterscheidung zwischen 
Tab. 1 Übliche Deskriptoren des Verhal-

tens während und nach einem Anfall (alphabetisch angeordnet)

\begin{tabular}{|c|c|}
\hline Kognitiv & Automatismen \\
\hline Akalkulie & Aggression \\
\hline Aphasie & Entkleiden \\
\hline $\begin{array}{l}\text { Aufmerksamkeits- } \\
\text { störung }\end{array}$ & Laufen \\
\hline $\begin{array}{l}\text { Beeinträchtige } \\
\text { Reagibilität }\end{array}$ & Lidflattern \\
\hline $\begin{array}{l}\text { Déjà vu oder } \\
\text { Jamais vu }\end{array}$ & Manuell \\
\hline Dissoziation & Nicken \\
\hline Gedächtnisstörung & Orofazial \\
\hline Halluzinationen & Perseveration \\
\hline Illusionen & Radfahren \\
\hline Neglekt & Rennen \\
\hline Zwangsgedanken & $\begin{array}{l}\text { Rhythmische Becken- } \\
\text { bewegungen }\end{array}$ \\
\hline $\begin{array}{l}\text { Emotional } \\
\text { oder affektiv }\end{array}$ & Sexuell \\
\hline Agitation & Vokalisation/Sprache \\
\hline Angst & Motorisch \\
\hline Furcht & Dysarthrie \\
\hline $\begin{array}{l}\text { Lachen } \\
\text { (gelastisch) }\end{array}$ & Dystonie \\
\hline Lust & Fechterstellung \\
\hline Wahn & Jackson-March \\
\hline $\begin{array}{l}\text { Weinen } \\
\text { (dakrystisch) }\end{array}$ & Koordinationsstörung \\
\hline Wut & Lähmung \\
\hline Autonom & Version \\
\hline Asystolie & Sensorisch \\
\hline Blässe & Auditorisch \\
\hline Bradykardie & Gustatorisch \\
\hline Erektion & Olfaktorisch \\
\hline Erröten & Somatosensibel \\
\hline Gastrointestinal & Temperaturempfindung \\
\hline Gänsehaut & Vestibulär \\
\hline $\begin{array}{l}\text { Hyper-/ } \\
\text { Hypoventilation }\end{array}$ & Visuell \\
\hline Palpitationen & Lateralisation \\
\hline $\begin{array}{l}\text { Respiratorische } \\
\text { Veränderungen }\end{array}$ & Links \\
\hline Tachykardie & Rechts \\
\hline $\begin{array}{l}\text { Übelkeit oder } \\
\text { Erbrechen }\end{array}$ & Bilateral \\
\hline
\end{tabular}

typischer und atypischer Absence bei, da die beiden Anfallsformen mit unterschiedlichen EEG-Korrelaten, Epilepsiesyndromen, Therapien und Prognosen einhergehen. Entsprechend der 1981erKlassifikation, die auf zahlreichen Vi-
deo-EEG-Aufnahmen beruhte, werden Absencen als atypisch eingestuft, wenn sie stärker als bei typischen Absencen mit Veränderungen im Muskeltonus assoziiert sind oder der Beginn oder das Ende nicht abrupt ist. Ein EEG kann erforderlich sein, um die Unterscheidung zwischen typischen und atypischen Absencen zu treffen.

Eine myoklonische Absence [9] bezeichnet eine Absence mit rhythmischen 3/s myoklonischen Bewegungen, die zu einer zunehmenden Armabduktion und -elevation führt und mit 3/s generalisierten Spike-wave-Entladungen assoziiert ist. Die Dauer beträgt typischerweise 10-60 s. Eine Beeinträchtigung des Bewusstseins ist oft nicht offensichtlich. Myoklonische Absencen treten sowohl bei einer Vielzahl genetischer Epilepsien wie auch ohne bekannte genetische Assoziationen auf.

Lidmyoklonien sind myoklonische Zuckungen der Augenlider mit Bulbusdeviation nach oben und werden häufig durch Augenschluss oder Licht provoziert. Lidmyoklonien sind häufig mit Absencen assoziiert, können jedoch auch rein motorische Anfälle ohne assoziierte Absence sein, was eine Kategorisierung erschwert. Die 2017er Klassifikation ordnet sie nichtmotorischen Absencen $\mathrm{zu}$, was kontraintuitiv erscheinen mag; die Myoklonien werden in diesem Fall jedoch als Absence-assoziiert aufgefasst und nicht als motorischer Anfall. Absencen mit Lidmyoklonien, (generalisiert tonisch-klonische) Anfälle sowie durch Lidschluss induzierte EEG-Paroxysmen und Fotosensitivität konstituieren die Triade des Jeavons-Syndroms [10].

Anfälle mit unbekanntem Beginn können motorisch oder nichtmotorisch sein. Die Hauptanwendung dieser Klassifizierung besteht für tonisch-klonische Anfälle mit unklarem Beginn. Zusatzinformationen können eine Reklassifizierung als fokal oder generalisiert beginnenden Anfall ermöglichen. Epileptische Spasmen und Anfälle mit Innehalten sind weitere mögliche Anfallsformen mit unklarem Beginn. Zur Klärung der Natur des Beginns epileptischer Spasmen kann ein detailliertes Video-EEG-Monitoring erforderlich sein; dessen Durchführung ist auch wichtig, weil ein fokaler Beginn auf eine behandelbare fokale Pathologie hinweisen kann. Ein Anfall mit unbekanntem Beginn und Innehalten könnte für einen fokalen Anfall mit Bewusstseinsstörung und Innehalten oder für eine Absence stehen. Ein Anfall kann auch aufgrund inadäquater Information oder der Unmöglichkeit, ihn in eine andere Kategorie einzuordnen, unklassifiziert bleiben. Wenn ein Ereignis nicht eindeutig ein epileptischer Anfall ist, sollte es nicht als unklassifizierter Anfall bezeichnet werden; diese Klassifikation ist reserviert für ungewöhnliche Ereignisse, die mit hoher Wahrscheinlichkeit epileptische Anfälle, jedoch ansonsten nicht charakterisiert sind.

Bei jeder Anfallsklassifizierung gibt es einen gewissen Grad an Unsicherheit. Die Task Force übernahm die allgemeine Richtlinie, dass man zu $80 \%$ sicher sein sollte, dass der Beginn fokal oder generalisiert ist; andernfalls sollte der Anfall als „mit unbekanntem Beginn“ gelistet werden. Die $80 \%$-Schwelle wurde arbiträr gewählt entsprechend der $80 \%$ Grenze für falsch negative Ergebnisse statistischer Analysen.

\section{Allgemeine Deskriptoren}

Fokale Anfälle bringen eine Vielzahl potenzieller Empfindungen und Verhaltensweisen hervor, die $\mathrm{zu}$ vielfältig sind, um sie in eine Klassifikation aufzunehmen. Zur Erleichterung einer gemeinsamen Terminologie für Anfälle hat die Taskforce einige verbreitete Deskriptoren für Verhalten während fokalen Anfällen aufgelistet (• Tab. 1); diese sind jedoch kein Bestandteil der Klassifikation. Anders ausgedrückt kann man diese allgemeinen Deskriptoren zur Anfallsklassifizierung hinzufügen, um die Manifestationen individueller Anfälle klar darzustellen, diese Deskriptoren definieren jedoch keine eigenständige Anfallsform in dieser Klassifikation. Deskriptoren stehen deshalb auf einer "niedrigeren Stufe“ als Zeichen wie „tonisch“, die eine Anfallsform definieren. Ein besonderer Deskriptor-Typ mit hoher Praxisrelevanz stellt die Lateralisierung dar. Die Task Force erkennt die Bedeutung detaillierter, individuel- 
Begriff

Absence, typische

Plötzlicher Beginn, Unterbrechung laufender Aktivitäten, starrer Blick, möglicherweise eine kurze

Adaptiert nach [11] Deviation der Augen nach oben. Meistens reagiert der Patient nicht auf Ansprache. Die Dauer beträg wenige Sekunden bis zu einer Minute mit sehr rascher Erholung. Wenn auch nicht immer verfügbar, würde ein EEG generalisierte epileptische Entladungen während des Vorkommnisses zeigen. Eine Absence ist per Definition ein Anfall mit generalisiertem Beginn. Das Word ist nicht mit "leerem Blick" synonym, den man auch bei fokal beginnenden Anfällen antrifft

Absence, atypische

Eine Absence mit Tonusänderung, die stärker ausgeprägt ist als bei einer typischen Absence, oder mit einem nicht abrupten Beginn oder Ende, oft assoziiert mit langsamer, irregulärer, generalisierter Spike-wave-Aktivität

Anfall

Ein vorübergehendes Auftreten von Symptomen aufgrund abnormer exzessiver oder synchronisierter neuronaler Hirnaktivität

Areagibel

Unfähig, angemessen mit Bewegung oder Sprache auf Stimuli zu reagieren

Atonisch

Plötzlicher Verlust oder Verminderung des Muskeltonus, ohne sichtbare vorangehende Myoklonie oder Tonisierung, der für ca. 1-2 s anhält; mit Einbeziehung von Kopf-, Rumpf-, Kiefer- oder Extremitätenmuskulatur

Automatismus

Eine mehr oder weniger koordinierte motorische Aktivität, die üblicherweise bei beeinträchtigte Kognition auftritt und für die meistens (aber nicht immer) eine Amnesie besteht. Er ähnelt häufig mutwilligen Bewegungen und kann eine inadäquate Fortsetzung präiktaler motorischer Aktivität beinhalten

Autonomer Anfall

Eine deutliche Änderung von Funktionen des autonomen Nervensystems, einschließlich kardiovaskulärer, pupillomotorischer, gastrointestinaler, sudomotorischer, vasomotorischer und thermoregulatorischer Funktionen

Aura

Ein subjektives, iktuales Phänomen, das einem beobachtbaren Anfall vorhergehen kann (Umgangssprache)

Bewusstheit

Wissen vom Selbst und der Umgebung

Adaptiert nach [12]

Adaptiert nach [11]

Bewusstsein

Ein Geisteszustand mit subjektiven und objektiven Aspekten, der Selbstbewusstsein, Bewusstheit, Reaktivität und Erinnerungsvermögen beinhaltet

Bewusstseinsgestört Kurzform für eine Störung des Bewusstseins

Bilateral

Sowohl linke als auch rechte Seite, wobei Manifestationen bilateraler Anfälle symmetrisch oder asymmetrisch sein können

Dakrystisch

Weinattacken, die mit Traurigkeit assoziiert sein können oder auch nicht

Dyston

Anhaltende Kontraktionen von agonistischen und antagonistischen Muskeln mit der Folge athetoider oder verdrehter Bewegungen, die abnormale Körperhaltungen bedingen können

Emotionale Anfälle

Anfälle mit Emotionen oder dem Anschein von Emotionen als frühes prädominantes Zeichen wie Angst, spontane Freude oder Euphorie, Lachen (gelastisch) oder Weinen (dakrystisch)

Epileptische Spasmen

Eine plötzliche Flexion, Extension oder gemischte Extension und Flexion vorwiegend der proximalen und Rumpfmuskulatur, die normalerweise anhaltender ist als eine Myoklonie, aber weniger anhaltend als ein tonischer Anfall. Limitierte Formen können auftreten: Grimassen, Nicken oder subtile Augenbewegungen. Epileptische Spasmen treten häufig in Clustern auf. Infantile Spasmen sind die bekannteste Form, aber Spasmen können in jedem Alter auftreten

Epilepsie

Eine Erkrankung des Gehirns, die durch mindestens eine der folgenden Bedingungen definiert wird: (I) Mindestens 2 unprovozierte oder Reflex-Anfälle innerhalb von weniger als $24 \mathrm{~h}$; (II) ein unprovozierter oder Reflex-Anfall und einer Wahrscheinlichkeit des Auftretens weiterer Anfälle entsprechend dem allgemeinen Wiederholungsrisiko $(>60 \%)$ nach 2 unprovozierten Anfällen innerhalb von 10 Jahren; (III) Vorliegen eines Epilepsiesyndroms. Eine Epilepsie wird als ausgeheilt eingestuft, wenn Individuen ein altersabhängiges Epilepsiesyndrom hatten, nun aber diesen Altersbereich verlassen haben oder wenn man die letzten 10 Jahre anfallsfrei war ohne Einnahme von Antikonvulsiva während der letzten 5 Jahre

Fechterstellung

Eine fokal motorische Anfallsform mit Extension eines Armes und Flexion des kontralateralen Ellenbogens und Handgelenkes, einem Schwertkampf ähnelnd. Dies wurde auch supplementär-motorischer Anfall genannt

Fokal

In auf eine Hemisphäre begrenzten Netzwerken entstehend. Diese können umschrieben lokalisiert oder weit ausgedehnt sein. Fokale Anfälle können in subkortikalen Strukturen entstehen

Fokal beginnender bilateral tonisch-klonischer Anfall

Eine Anfallsform mit fokalem Beginn, mit erhaltenem oder gestörtem Bewusstsein, motorisch oder nichtmotorisch, der in bilateral tonisch-klonische Aktivität übergeht. Die frühere Bezeichnung war Anfall mit partiellem Beginn und sekundärer Generalisierung

\section{[11]}

$\mathrm{Neu}$

$\mathrm{Neu}$

Adaptiert nach [11]

$\mathrm{Neu}$

Adaptiert nach [11] 


\section{Standards und Leitlinien}

\section{Tab. 2 (Fortsetzung)}

\begin{tabular}{|c|c|}
\hline Begriff & Definition \\
\hline Gelastisch & Lachanfälle oder Kichern, meist ohne entsprechende affektive Tönung \\
\hline Generalisiert & An irgendeinem Punkt eines bilateralen Netzwerkes entstehend mit rascher Ausbreitung \\
\hline $\begin{array}{l}\text { Generalisiert to- } \\
\text { nisch-klonisch }\end{array}$ & $\begin{array}{l}\text { Bilateral symmetrisch oder manchmal asymmetrische tonische Kontraktion und dann bilateral kloni- } \\
\text { sche Kontraktionen der Muskulatur, meist assoziiert mit autonomen Phänomen und Bewusstseins- } \\
\text { verlust. Diese Anfälle involvieren von Beginn an Netzwerke in beiden Hemisphären }\end{array}$ \\
\hline $\begin{array}{l}\text { Gestörtes Bewusst- } \\
\text { sein }\end{array}$ & $\begin{array}{l}\text { Siehe Bewusstheit. Ein gestörtes oder verlorenes Bewusstsein ist eine Eigenschaft von fokalen Anfäl- } \\
\text { len mit Bewusstseinsstörung, früher „komplex partielle Anfälle" genannt }\end{array}$ \\
\hline Halluzination & $\begin{array}{l}\text { Eine Bildung zusammengesetzter Wahrnehmungen ohne entsprechende externe Stimuli, mit vi- } \\
\text { suellen, auditorischen, somatosensiblen, Geruchs- oder/auch Geschmacksphänomenen. Beispiel: } \\
\text { Personen sprechen „hören“ oder "sehen“ }\end{array}$ \\
\hline Immobilität & Siehe [Innehalten] \\
\hline
\end{tabular}

Innehalten

Arrest (Pause) von Aktivitäten, Erstarren, Immobilisation, wie bei einem Anfall mit Innehalten

Quelle

Jackson-Anfall

Traditionelle Bezeichnung, die die unilaterale Ausbreitung klonischer Bewegungen über benachbarte Körperregionen anzeigt

Klonisch

Symmetrische oder asymmetrische Zuckungen, die rhythmisch sind und identische Muskelgruppen betreffen

Kognitiv

Das Denken oder höhere kortikale Funktionen betreffend, wie Sprache, räumliche Wahrnehmung,

Erinnerungsvermögen, Praxis. Der frühere Begriff zur gleichen Verwendung als Anfallsform war "psychisch"

Lidmyoklonie

Zuckungen des Augenlids mit einer Frequenz von mindestens 3/s, meist mit Augendeviation nach oben, meist weniger als $10 \mathrm{~s}$ dauernd, oft ausgelöst durch Augenschluss. Dies kann, muss jedoch nicht, mit einem kurzem Bewusstheitsverlust verbunden sein

Störung des Bewusstseins

Siehe gestörtes Bewusstsein

Motorisch

Einbeziehung der Muskulatur in jeglicher Form. Das motorische Ereignis kann in einer Zunahme (positiv) oder in einer Minderung (negativ) der Muskelkontraktion und der sich hieraus entwickelnden Bewegung bestehen

Myoklonisch

Plötzliche, kurze (<100 ms), unwillkürliche einzelne oder mehrfache Kontraktionen von Muskeln oder Muskelgruppen variabler Topographie (axial, proximale oder distale Extremitäten, distal). Myoklonien sind weniger regelmäßig repetitiv und weniger anhaltend als Kloni

Myoklonisch-atonisch Eine generalisierte Anfallsform mit einer Myoklonie, die in einen atonischen motorischen Abschnitt übergeht. Diese Form wurde früher myoklonisch-astatisch genannt

Myoklonisch-tonisch- Eine oder wenige bilaterale Zuckungen der Gliedmaßen, gefolgt von einem tonisch-klonischen Anklonisch fall. Die anfänglichen Zuckungen können als kurze Periode von Kloni oder Myoklonien betrachtet werden. Diese Anfälle kommen häufig bei juveniler myoklonischer Epilepsie vor

Nichtmotorisch Fokale oder generalisierte Anfallsformen ohne prominente motorische Aktivität

[11]

[4]

Adaptiert nach $[4$ 11]

$\mathrm{Neu}$

$\mathrm{Neu}$

[11]

Adaptiert nach [11]

$\mathrm{Neu}$

$\mathrm{Neu}$

Adaptiert nach [11]

$\mathrm{Neu}$

Abgeleitet aus [12]

$\mathrm{Neu}$

$\mathrm{Neu}$ weiterer Hirnnetzwerke

Reagibilität

Die Fähigkeit, angemessen mit Bewegung oder Sprache auf Stimuli zu reagieren

Sensorischer Anfall

Eine Wahrnehmung, die nicht durch adäquate Stimuli der Außenwelt verursacht ist

Spasmus

Siehe epileptische Spasmen

Tonisch

Eine anhaltende Zunahme der Muskelkontraktion, die einige Sekunden bis Minuten anhält

Eine Sequenz aus einer tonischen, gefolgt von einer klonischen Phase

Unklassifiziert

Bezieht sich auf eine Anfallsform, die nicht anhand der ILAE-Klassifikation von 2017 beschrieben werden kann aufgrund unzureichender Information oder ungewöhnlicher klinischer Merkmale. Wenn der Anfall unklassifiziert ist, weil die Art des Beginns unbekannt ist, kann dennoch eine limitierte Klassifikation aus den beobachteten Merkmalen abgeleitet werden

Verhaltensarrest

Siehe "Innehalten“

Version

Eine anhaltende, forcierte konjugierte Blickbewegung, Kopf- oder Rumpfrotation oder Bewegung zur Seite

"Zeichen der Zahl 4" Extension eines Armes (meistens kontralateral zu der epileptogenen Zone) mit Ellenbogenflexion des Neu anderen Arms, das Bild einer 4 bildend

Neu neue Definition, im vorliegenden Beitrag erstellt 
Tab. 3 Gegenüberstellung alter und neuer Begriffe zur Anfallsklassifizierung

\begin{tabular}{|c|c|}
\hline Alte Anfallsterminologie & Neue Anfallsterminologie \\
\hline Absence & (Generalisierte) Absence \\
\hline Absence, typische & (Generalisierte) Absence, typische \\
\hline Absence, atypische & (Generalisierte) Absence, atypische \\
\hline Akinetisch & Fokaler Anfall mit Innehalten, generalisierte Absence \\
\hline Astatisch & [Fokal/generalisiert] atonisch \\
\hline Atonisch & [Fokal/generalisiert] atonisch \\
\hline Aura & Bewusst erlebt fokal \\
\hline Dakrystisch & [Bewusst erlebt/nicht bewusst erlebt] fokal-emotional (dakrystisch) \\
\hline Dialeptisch & Nicht bewusst erlebt fokal \\
\hline Einfach partiell & Bewusst erlebt fokal \\
\hline Fechterstellung (asymmetrisch tonisch) & [Bewusst erlebt/nicht bewusst erlebt] fokal motorisch tonisch \\
\hline Innehalten & [Bewusst erlebter/nicht bewusst erlebter] fokaler Verhaltensarrest \\
\hline Frontallappen[-Anfall] $]^{\mathrm{a}}$ & Fokal \\
\hline Gelastisch & [Bewusst erlebt/nicht bewusst erlebt] fokal emotional (gelastisch) \\
\hline Grand mal & $\begin{array}{l}\text { Generalisiert tonisch-klonisch, fokal (mit Übergang) zu bilateral-tonisch-klonisch, } \\
\text { tonisch-klonisch mit unbekanntem Beginn }\end{array}$ \\
\hline Gustatorisch & [Bewusst erlebt/nicht bewusst erlebt] fokal sensorisch (gustatorisch) \\
\hline Infantile Spasmen & [Fokal/generalisiert/unbekannt beginnende] epileptische Spasmen \\
\hline Jackson[-Anfall] & Bewusst erlebt fokal-motorisch (Jackson-Anfall) \\
\hline Limbisch & Nicht bewusst erlebt fokal \\
\hline Großer Anfall & $\begin{array}{l}\text { Generalisiert tonisch-klonisch, fokal (mit Übergang) zu bilateral tonisch-klonisch, to- } \\
\text { nisch-klonisch mit unbekanntem Beginn }\end{array}$ \\
\hline Kleiner Anfall mit motorischer Komponente & Fokal motorisch, generalisiert myoklonisch \\
\hline Klonisch & [Fokal/Generalisiert] klonisch \\
\hline Komplex partiell & Nicht bewusst erlebt fokal \\
\hline Konvulsion & $\begin{array}{l}\text { [Fokal/generalisiert] motorisch [tonisch-klonisch, tonisch, klonisch], fokal mit Übergang } \\
\text { in bilateral tonisch-klonisch }\end{array}$ \\
\hline Myoklonisch & [Fokal/generalisiert] myoklonisch \\
\hline Neokortikal ${ }^{\mathrm{a}}$ & Bewusst erlebt/nicht bewusst erlebt fokal \\
\hline Okzipitallappen[-Anfall $]^{a}$ & Fokal \\
\hline Parietallappen[-Anfall $]^{\mathrm{a}}$ & Fokal \\
\hline Partiell & Fokal \\
\hline Petit mal & Absence \\
\hline Psychomotorisch & Nicht bewusst erlebt fokal \\
\hline Rolandisch & Bewusst erlebt fokal motorisch, fokal (mit Übergang) zu bilateral tonisch-klonisch \\
\hline Salaam & [Fokal/generalisiert/unbekannt beginnender] epileptischer Spasmus \\
\hline Sekundär generalisiert tonisch-klonisch & Fokal (mit Übergang) zu bilateral tonisch-klonisch \\
\hline Sturzanfall & [Fokal/generalisiert] atonisch, [fokal/generalisiert] tonisch \\
\hline Supplementär-motorisch & Fokal motorisch tonisch \\
\hline Sylvisch & Fokal motorisch \\
\hline Temporallappen[-Anfall $]^{\mathrm{a}}$ & Bewusst erlebt/Nicht bewusst erlebt fokal \\
\hline Tonisch & [Fokal/generalisiert] tonisch \\
\hline Tonisch-klonisch & $\begin{array}{l}\text { [Generalisiert/unbekannt] beginnend tonisch-klonisch, fokal (mit Übergang) zu } \\
\text { bilateral tonisch-klonisch }\end{array}$ \\
\hline Uncinatuskrise & Bewusst erlebt/nicht bewusst erlebt fokal-sensorisch (olfaktorisch) \\
\hline „Zeichen der 4“ & [Bewusst erlebt/nicht bewusst erlebt] fokal motorisch tonisch \\
\hline \multicolumn{2}{|c|}{ Zu beachten ist, dass keine Eins-zu-eins-Entsprechung besteht, da die Umbenennung mit einer Reorganisation einhergeht. Die wichtigsten Begriffe sind } \\
\hline
\end{tabular}


Tab. 4 Abkürzungen für die häufigsten wichtigen Anfallsformen

Anfallsform

Abkür-

zung

Bewusst erlebter fokaler Anfall

Nicht bewusst erlebter fokaler

Anfall

Fokaler motorischer Anfall

Fokaler nichtmotorischer Anfall

Fokale epileptische Spasmen

Fokaler zu bilateral tonisch-kloni-

scher Anfall

Generalisierter tonisch-klonischer

Anfall

Generalisierter Absenceanfall

Generalisierter motorischer Anfall

Generalisierte epileptische Spas-

men

Unbekannt beginnender tonisch

klonischer Anfall

ler Freitextbeschreibungen eines Anfalls zusätzlich zur Klassifizierung an.

- Tab. 4 listet Abkürzungsvorschläge für die Hauptanfallsformen auf.

\section{Zusammenfassung der Regeln für das Klassifizieren von Anfällen}

1. Beginn: Mit mindestens $80 \%$ iger Sicherheit entscheiden, ob der Beginn fokal oder generalisiert ist. Wenn dies nicht möglich ist, ist der Beginn unbekannt.

2. Bewusstsein: Bei fokalen Anfällen, entscheiden, ob man anhand des Grades an Bewusstheit klassifiziert, oder ob Bewusstheit als Klassifikator weggelassen wird. Bewusst erlebte fokale Anfälle entsprechen den bisherigen einfach-partiellen Anfällen und nicht bewusst erlebte fokale Anfälle den bisherigen komplexen-partiellen Anfällen.

3. Beeinträchtigtes Bewusstsein an irgendeinem Punkt: Ein fokaler Anfall gilt als Anfall mit Bewusstseinsbeeinträchtigung, wenn das Bewusstsein zu irgendeinem Punkt des Anfalls beeinträchtigt ist.

4. Prädominanter Beginn: Klassifizierung eines fokalen Anfalls anhand des ersten prominenten Symptoms.
5. Innehalten: Ein fokaler Anfall mit Innehalten zeigt ein Sistieren von Bewegungen als das prominente Merkmal des gesamten Anfalls.

6. Motorisch/nichtmotorisch: Ein bewusst erlebter fokaler oder nicht bewusst erlebter fokaler Anfall kann anhand motorischer oder nicht motorischer Charakteristika weiter subklassifiziert werden.

Alternativ kann ein fokaler Anfall mit motorischen oder nichtmotorischen Charakteristika beschrieben werden, ohne den Bewusstseinszustand weiter zu spezifizieren. Beispiel: ein fokal tonischer Anfall.

7. Optionale Termini: Termini wie motorisch oder nichtmotorisch können weggelassen werden, wenn die Anfallsform ansonsten unmissverständlich ist.

8. Zusätzliche Deskriptoren: Nach Klassifizierung der Anfallsform anhand initialer Manifestationen wird befürwortet, andere Zeichen oder Symptome anhand von Deskriptoren oder Freitext weiter zu beschreiben. Diese verändern nicht die Anfallsform. Beispiel: Fokal emotionaler Anfall mit tonischer Bewegung des rechten Armes und Hyperventilation.

9. Bilateral vs. generalisiert: Die Bezeichnung „bilateral“ wird für tonisch-klonische Anfälle genutzt, die sich auf beide Hemisphären ausbreiten; die Bezeichnung "generalisiert“ für Anfälle, die augenscheinlich gleichzeitig in beiden Hemisphären entstehen.

10. Atypische Absence: Eine Absence ist atypisch, wenn sie einen langsamen Beginn oder ein allmähliches Ende hat, eine deutliche Tonusänderung oder einen EEG-Anfallsbeginn mit Spike-wave-Komplexen einer Frequenz von $<3 /$ s aufweist.

11. Klonisch vs. myoklonisch: Klonisch bedeutet anhaltendes rhythmisches Zucken und myoklonisch regelmäßiges, jedoch nicht anhaltendes Zucken.

12. Lidmyoklonie: Absence mit Lidmyoklonien bedeutet forciertes Aufwärtszucken der Augenlider während einer Absence.

\section{Glossar}

- Tab. 2 bietet ein Glossar der Termini, die in diesem und dem begleitenden Text verwendet werden. Die Definitionen sind nicht universal, aber fokussieren sich auf die Aspekte der Fachsprache, mit der Anfälle beschrieben werden. Beispielsweise ist „sensorisch“ in Bezug auf sensorische Anfälle definiert, nicht auf alle Wahrnehmungen. Wenn möglich, wurden zur Unterstützung der Kontinuität früher akzeptierte Definitionen des ILAE-Glossars von 2001 [11] beibehalten, aber dieses Glossar aktualisiert einige Termini. Zur Definition alter Termini kann die ältere Literatur hinzugezogen werden. Termini, die nicht länger empfohlen werden, wurden weggelassen.

\section{Gegenüberstellung alter und neuer Termini}

- Tab. 3 bietet eine Gegenüberstellung alter offizieller und gebräuchlicher Termini zur Klassifikation der Anfallsformen von 2017.

\section{Beispiele}

1. Tonisch-klonisch: Eine Frau erwacht und findet ihren Ehemann vor, während er im Bett einen Anfall hat. Der Beginn wird nicht beobachtet, aber sie kann eine bilaterale Versteifung, gefolgt von bilateralen Zuckungen beschreiben. EEG und Magnetresonanztomographie (MRT)-Befunde sind normal.

Dieser Anfall wird als tonischklonisch mit unklarem Beginn klassifiziert. Es gibt keine ergänzenden Informationen, die erlauben würden, den Beginn als fokal oder generalisiert zu bestimmen. In der alten Klassifikation wäre dieser Anfall unklassifizierbar ohne weitere Vermerke gewesen.

2. Bilateral tonisch-klonisch mit fokalem Beginn: In einem alternativen Szenario zu Fall 1 zeigt das EEG einen klaren rechts parietalen Verlangsamungsherd. Magnetresonanztomographisch zeigt sich eine kortikale Dysplasie rechts parietal. 
Unter diesen Umständen kann der Anfall als bilateral tonisch-klonisch mit fokalem Beginn klassifiziert werden trotz des nicht beobachteten Beginns, da eine fokale Ätiologie identifiziert wurde und der Anfall mit überwältigender Wahrscheinlichkeit einen fokalen Beginn hatte. Die alte Klassifikation hätte diesen Anfall als partiellen Anfall mit sekundärer Generalisierung klassifiziert.

3. Absence: Bei einem Kind wird ein Lennox-Gastaut-Syndrom unbekannter Ätiologie diagnostiziert. Das EEG zeigt Züge von Slowspike-Waves. Anfallsformen umfassen Absencen, tonische und fokal-motorische Anfälle. Die Absencen verlaufen prolongiert mit undeutlich abgegrenztem Beginn und Ende und führen manchmal zu Stürzen. In diesem Fall werden die Absencen als atypisch klassifiziert aufgrund ihrer Charakteristika, des EEG-Musters und des zugrunde liegenden Syndroms. Die Absencen wären im alten System in gleicher Weise klassifiziert worden.

4. Tonisch: Ein Kind hat kurze Anfälle mit Versteifung des rechten Arms und Beins, während derer Reaktivität und Bewusstsein erhalten bleiben. Dieser Anfall ist ein bewusst erlebter fokaler tonischer Anfall mit erhaltenem Bewusstsein (der Zusatz „motorischer Beginn“ ist implizit). Im alten System hätte man diesen Anfall tonisch genannt, evtl. in fälschlicher Annahme eines generalisierten Beginns.

5. Nicht bewusst erlebt fokal: Eine 25 Jahre alte Frau beschreibt Anfälle, die mit einem 30-sekündigem, intensiven Gefühl beginnen, dass „bekannte Musik spielt“. Sie kann andere Personen sprechen hören, erkennt jedoch im Nachhinein, dass sie nicht verstehen konnte, was sie sagten. Nach einer Episode ist sie leicht verwirrt und muss „sich neu orientieren“. Der Anfall würde als fokal mit Bewusstseinsstörung klassifiziert. Obwohl die Patientin in der Lage ist, mit ihrer Umgebung zu interagieren, kann sie sie nicht verstehen und ist leicht verwirrt. Die frühere Klassifikation wäre ein komplex-partieller Anfall gewesen

6. Autonom: Ein 22-jähriger Mann hat Anfälle, die er vollkommen bewusst erlebt, mit Gänsehaut an den Armen und einem Hitzegefühl. Diese werden als fokale nichtmotorische autonome Anfälle mit erhaltenem Bewusstsein klassifiziert oder prägnanter als bewusst erlebte fokal autonome Anfälle. Die alte Klassifizierung hätte sie einfach partiell autonome Anfälle genannt.

7. Fokal klonisch: Ein 1 Monat alter Junge hat rhythmische Zuckungen des linken Armes, die bei Umlagerung des Armes nicht aufhören. Ein korrespondierendes EEG zeigt rechts frontale iktale Rhythmen. Diese Anfälle sind klonische Anfälle fokal motorischen Beginns, oder kürzer, fokale klonische Anfälle. Da der Bewusstseinszustand nicht feststellbar ist, wird die Bewusstseinslage bei der Klassifikation nicht berücksichtigt. Die alte Klassifikation hätte für diesen Anfall keine Bezeichnung gehabt.

8. Sequenzielle Anfallsmanifestationen: Ein Anfall beginnt mit Kribbeln im rechten Arm eines 75-jährigen Mannes. Der Patient sagt, es gehe dann in rhythmisches Zucken des rechten Arms über, das etwa $30 \mathrm{~s}$ lang anhalte. Er behält Bewusstsein und Erinnerungsfähigkeit während des Vorkommnisses. Dieser Anfall ist ein (bewusst erlebter) fokaler sensorischer Anfall mit nichtmotorischem Beginn. Eine zusätzliche Beschreibung wäre nützlich, namentlich fokal sensorischer Anfall mit somatosensorischen Eigenschaften, der übergeht in Kloni im rechten Arm. Wenn die sensorischen und motorischen Vorkommnisse diskontinuierlich wären oder der Arzt Grund hätte, das Vorkommnis als 2 separate (bifokale oder multifokale) Anfälle einzuschätzen, dann würde jede Komponente als ein separater Anfall klassifiziert werden. Die alte Klassifikation hätte dies einen einfach-partiellen senso- motorischen Anfall genannt. Ein Vorteil der 2017er-Klassifikation ist die Spezifikation des sensorischen Beginns, der klinische Relevanz haben kann.

9. Myoklonisch-atonisch: Ein 4 Jahre alter Junge mit Doose-Syndrom hat Anfälle mit ein paar Armzuckungen und raschem Tonusverlust. Diese werden neu als myoklonischatonische Anfälle klassifiziert. Im vorherigen inoffiziellen Gebrauch hätte man diese myoklonischastatische Anfälle genannt.

10. Myoklonisch-tonisch-klonische Anfälle: Ein 13-Jähriger mit juveniler myoklonischer Epilepsie hat Anfälle, die mit einigen Zuckungen beginnen, gefolgt von Versteifung der Gliedmaßen und daraufhin rhythmischem Zucken aller Extremitäten. Diese werden neu als myoklonisch-tonisch-klonische Anfälle klassifiziert. In der alten Klassifikation existierte keine spezielle Anfallsform, aber man hätte sie myoklonische oder tonische Anfälle, gefolgt von tonisch-klonischen Anfällen nennen können.

11. Fokale epileptische Spasmen: Ein 14 Monate altes Mädchen erleidet plötzliche Extension beider Beine und Rumpfflexionen für etwa $2 \mathrm{~s}$ Dauer. Diese Anfälle wiederholen sich in Clustern. Der EEG zeigt eine Hypsarrhythmie mit bilateralen Spikes mit Betonung links parietal. Das MRT zeigt eine links parietale Dysplasie, eine Resektion der Dysplasie führt zu Anfallsfreiheit. Aufgrund der Zusatzinformation würde man die Anfallsform als fokal epileptische Spasmen einschätzen (die Bezeichnung motorischer Beginn ist implizit). Die vorherige Klassifizierung hätte dies infantile Spasmen genannt ohne Information zur Fokalität. Die Bezeichnung „infantil“ kann weiterhin genutzt werden, wenn Spasmen in der Kindheit auftreten.

12. Unklassifiziert: Ein 75 Jahre alter Mann mit Epilepsie berichtet von einem inneren Gefühl von Körperzittern und einem Gefühl von Verwirrung. Keine weiteren 
Informationen sind verfügbar. EEG und MRI sind normal. Dieses Ereignis ist unklassifiziert.

\section{Diskussion}

Dieser Begleittext zu dem Text, der die Gedanken und Struktur der Anfallsklassifikation der ILAE 2017 darstellt, bietet anwendungsorientierte Hinweise zur Nutzung der Klassifikation. Aber auch umfangreiche Erklärungen können die inhärenten Mehrdeutigkeiten einer Klassifikation im klinischen Alltagsgebrauch nicht eliminieren. Zum Beispiel können generalisiert beginnende tonisch-klonische Anfälle leicht asymmetrisch mit initialer Kopfversion verlaufen. Wie fokal muss eine Asymmetrie sein, um einen fokalen Beginn zu implizieren? Die Antwort liegt in der individuellen Beurteilung jedes Anfalls. Wie unsicher muss ein Arzt über die Beschaffenheit eines Beginns sein, um einen Anfall mit „unbekanntem Beginn“ zu klassifizieren? Die Taskforce setzte eine $80 \%$ Sicherheit als Richtlinie, um einen Anfall als fokal oder generalisiert zu bezeichnen, aber zweifelsohne wird diese Grenze in der Praxis unscharf sein.

Mehrdeutigkeiten kommen vor, wenn ein Anfall mehrere Symptome hat, beispielsweise Tonisierung des Armes und Automatismen. Der Klassifizierende sollte das früheste, prominente Symptom wählen, aber verschiedene Beobachter mögen verschiedene Anfallsbezeichnungen wählen in Abhängigkeit von der Interpretation berichteter oder beobachteter Symptome. Diese Mehrdeutigkeiten können teilweise vermindert werden, wenn man die typischen Muster üblicher Anfälle kennt. Ein Innehalten, gefolgt von Lidflattern und Nicken für $5 \mathrm{~s}$ und sofortiger Erholung ist wahrscheinlich eine typische Absence, auch wenn jedes einzelne Symptom in mehreren Anfallsformen vorkommen kann. Zur Anfallsform hinzugefügte, optionale Deskriptoren können mehr über die Natur des Anfalls aussagen, beispielsweise, wenn man „mit Lachen“ zu „fokaler emotionaler Anfall mit Bewusstseinsstörung" hinzufügt.

Diverse motorische Zeichen erscheinen jetzt in Verbindung mit fokalen oder generalisiert beginnenden Anfallsformen, aber es kann nicht davon ausgegangen werden, dass die Pathophysiologie bei beiden Kategorien identisch ist. Ein fokal tonischer Anfall kann andere Mechanismen als ein generalisierter tonischer Anfall haben, und jede Anfallsform kann verschiedene Prognosen, Ansprechen auf Behandlung, Demografie und Assoziationen mit Epilepsiesyndromen beinhalten. Selbst innerhalb der Kategorie fokal kann eine fokale Tonisierung als Teil eines fokalen Anfalls mit Bewusstseinsbeeinträchtigung (man denke an die häufige Dystonie während nicht bewusst erlebter fokaler Anfälle) eine andere Entität sein als bei fokal tonischen Anfällen bei einem Kind mit Lennox-Gastaut-Syndrom. Die Identifizierung dieser neuen Anfallsformen sollte es ermöglichen, mehr über sie und die mit ihnen assoziierten Syndrome zu lernen.

Eine Lern- und Akzeptanzkurve wird sich für diejenigen in der Gemeinschaft der Epileptologen entwickeln, die die 2017er-Klassifikation benutzen. Im Laufe der Zeit wird sich ein Konsens darüber herausbilden, welche Anfallsformen am besten die unterschiedlichen, wichtigen Symptomgruppen widerspiegeln. Die vergangenen Erfahrungen legen eine graduelle Akzeptanz der neuen Klassifizierung nahe mit kurzzeitiger Benutzung von Termini aus früheren Generationen von Klassifikationen. Die Anwendung der 2017er-Klassifikation im Alltag wird wahrscheinlich zu Revisionen führen. Eine erwünschte Auswirkung der 2017erKlassifikation ist ein vereinfachter Austausch über Anfallsformen unter Ärzten, der nichtmedizinischen Gemeinschaft und Forschern. Künftige empirische Klassifikationen werden entwickelt werden, wenn das Wissen für die Konstruktion einer Klassifikation, basierend auf fundamentalen Ursachen der unterschiedlichen Anfallsformen, ausreicht.

\section{Korrespondierender Übersetzer}

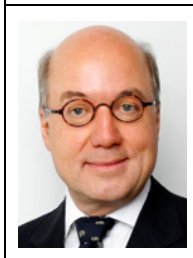

Prof. Dr. med. Andreas Schulze-Bonhage Epilepsiezentrum

Universitätsklinikum Freiburg

Neurozentrum

Breisacher Str. 64

79106 Freiburg

Deutschland

andreas.schulze-bonhage@

uniklinik-freiburg.de

\section{Korrespondenzadresse}

\section{Robert S. Fisher}

Neurology, SNHC

Room 4865, 213 Quarry Road, 94304 Palo Alto, CA, USA

robert.fisher@stanford.edu

\section{Einhaltung ethischer Richtlinien}

Interessenkonflikt. R. S. Fisher besitzt Aktienoptionen von Avail Pharmaceuticals, Cerebral Therapeutics, Zeto und Smart Monitor und hat Forschungszuschüsse von Medtronic und der National Science Foundation (NSF) erhalten. J. A. French gibt Unterstützung durch das Epilepsy Study Consortium an, das ihren universitären Arbeitgeber für ihre Beratertätigkeit im Zusammenhang mit Acorda, Alexza, Anavex, BioPharm Solutions, Concert, Eisai, Georgia Regents University, GW Pharma, Marathon, Marinus, Neurelis, Novartis, Pfizer, Pfizer-Neusentis, Pronutria, Roivant, Sage, SciFluor SK Life Sciences, Takeda, Turing, UCB Inc., Ultragenyx Upsher Smith, Xenon Pharmaceuticals und Zynerba bezahlt, darüber hinaus Zuschüsse und Forschungsunterstützung von Acorda, Alexza, LCGH, Eisai Medical Research, Lundbeck, Pfizer, SK Life Sciences, UCB, Upsher-Smith und Vertex sowie Forschungsunterstützung durch das National Institute of Neurological Disorders and Stroke (NINDS), das Epilepsy Therapy Project, die Epilepsy Research Foundation, und das Epilepsy Study Consortium. Sie erhielt eine Bezahlung als Mitglied des Editorial Boads von Lancet Neurology, Neurology Today und Epileptic Disorders und frühere Tätigkeit als Associate Editor von Epilepsia. S. R. Haut ist Beraterin für Acorda und Neurelis. E. Hirsch hat Honorare für Vorträge und / oder Beratung von Novartis, Eisai und UCB erhalten. S. L. Moshé eine jährliche Vergütung von Elsevier für seine Arbeit als Associate Editor für "Neurobiology of Disease", Tantiemen aus zwei Büchern, die er mit herausgegeben hat und Beraterhonorare von Eisai und UCB. J. Peltola hat an klinischen Studien für Eisai, UCB und Bial teilgenommen, erhielt Forschungsunterstützungen von Eisai, Medtronic, UCB und Cyberonics sowie Honorare von Cyberonics, Eisai, Medtronic, Orion Pharma und UCB, Reisekostenunterstützungen zu Kongressen von Cyberonics, Eisai, Medtronic und UCB und Honorare für Beratungstätigkeiten für Cyberonics, 
Eisai, Medtronic, UCB und Pfizer. I. E. Scheffer ist Mitglied der Editorial Boards von Neurology und Epileptic Disorders, erhält zukünftig möglicherweise Einnahmen aus dem schwebenden Patent WO61 / 010176 (eingereicht: 2008): therapeutische Substanz, und hat Beratungshonorare von GlaxoSmithKline, Athena Diagnostics, UCB, Eisai und Transgenomics erhalten. S. M. Zuberi ist Chefredakteur des European Journal of Pediatric Neurology, für das er ein jährliches Honorar von Elsevier Ltd. erhält, und erhielt Forschungsgelder von Dravet Syndrome UK, Epilepsy Research UK, UCB Pharma und der Glasgow Children's Hospital Charity. Dieverbleibenden Autoren listeten keine Angaben auf, die für die Klassifizierung von Anfallstypen relevant sind. C. D'Souza, E. Somerville und E. M. Yacubian haben keine Interessenkonflikte. A. Schulze-Bonhage hat Honorare für Vorträge und Beratung von Cyberonics, Desitin, Eisai, Precisis und UCB erhalten. M. Sperling hat Verträge über die Thomas Jefferson University mit Eisai, UCB Pharma, Sunovion, SK Life Sciences, Marinus, Lundbeck, Medtronics, Visualase, Accorda, Upsher-Smith, Brain Sentinel, und Glaxo geschlossen; er bekommt Forschungsunterstützung von der Defense Advanced Research Projects Agency (DARPA) und dem NIH über die Thomas Jefferson University. Zudem besteht ein Beratervertrag über die Thomas Jefferson University mit Medtronics. Wir bestätigen, dass wir die Position der Zeitschrift zu Themen, die mit ethischen Veröffentlichungen zu tun haben, gelesen haben und bestätigen und dass dieser Bericht mit diesen Leitlinien übereinstimmt.

Dieser Beitrag beinhaltet keine von den Autoren durchgeführten Studien an Menschen oder Tieren.

\section{Literatur}

1. Acharya JN, Wyllie E, Luders HO et al (1997) Seizure symptomatology in infants with localizationrelated epilepsy. Neurology 48:189-196

2. Fisher RS, Acevedo C, Arzimanoglou A et al (2014) A practical clinical definition of epilepsy. Epilepsia 55:475-482

3. Fisher RS, Boas WV, Blume W et al (2005) Epileptic seizures and epilepsy: definitions proposed by the International League against Epilepsy (ILAE) and the International Bureau for Epilepsy (IBE). Epilepsia 46:470-472

4. Berg AT, Millichap JJ (2013) The 2010 revised classification of seizures and epilepsy. Continuum (Minneap Minn) 19:571-597

5. Felician O, Tramoni E, Bartolomei F (2015) Transient epileptic amnesia: update on a slowly emerging epileptic syndrome. Rev Neurol (Paris) 171:289-297

6. Luat AF, Kamat D, Sivaswamy L (2015) Paroxysmal nonepileptic events in infancy, childhood, and adolescence. Pediatr Ann 44:e18-e23

7. WolfP, Yacubian EM, Avanzini Getal (2015) Juvenile myoclonic epilepsy: a system disorder of the brain. Epilepsy Res 114:2-12

8. Kelley SA, Kossoff EH (2010) Doose syndrome (myoclonic-astatic epilepsy): 40 years of progress. Dev Med Child Neurol 52:988-993

9. Elia M, Guerrini R, Musumeci SA et al (1998) Myoclonic absence-like seizures and chromosome abnormality syndromes. Epilepsia 39:660-663

10. Striano S, Capovilla G, Sofia V et al (2009) Eyelid myoclonia with absences (Jeavons syndrome): a well-defined idiopathic generalized epilepsy syndrome or a spectrum of photosensitive conditions? Epilepsia 50(Suppl.5):15-19
11. BlumeWT,Luders HO,MizrahiEetal (2001)Glossary of descriptive terminology for ictal semiology: report of the ILAE task force on classification and terminology. Epilepsia 42:1212-1218

12. Bancaud J et al (1981) Proposal for revised clinical and electroencephalographic classification of epileptic seizures. From the Commission on Classification and Terminology of the International League Against Epilepsy. Epilepsia 22:489-501 